\title{
Identification of Apple, orange and berry and segregation according to sizes in images using Image processing techniques
}

\author{
Ayushi Jain, Anubhav Srivastava
}

\begin{abstract}
Computer vision yields high level understanding by computers, one of the most important areas in recognition is object recognition which is the process of finding a specific object in an image or video sequence. In this paper we present an application that employs a part of object recognition, this application is able to distinguish between different kinds of fruits.The paper is intended to demonstrate the process by taking three fruit examples which can be further extended to greater number of fruit identification in an image.This application is based on color and size through comparing the fruit identified in image with predefined size of fruits for proper segregation of fruits. The paper can find immense use conveyor belt implementation for proper identification of size of fruit.
\end{abstract}

Index Terms - Image Processing; Object Recognition, OpenCV, Python

\section{INTRODUCTION}

Object recognition is the process of finding a specific object in an image or video sequence. It has became an important application of image processing, and have attracted the attention of many programmers recently. The paper describes a brief process to identify fruits which can be implemented on conveyor belt autonomous sorting of fruit.Further it can be used for educational purpose to enhance learning, especially for small kids and Down syndrome patients, of fruits pattern recognition and fruits features classification based on the fruit recognition result.Further the paper It can be used as a fruit recognition system in grocery store to automate labeling and computing the price. Our implementation included five steps:

(1) Learning process.

(2) Capture an image.

(3) Identify the fruit using color segmentation technique.

(4) Compare the image of the fruit so identified using step (3) with the image used for categorisation.

(5) Highlight the fruit so identified and thus send necessary data to the user.

These steps is illustrated below and depicted in (Figure 2).The rest of this paper is structured as follows:

Section two: provide a background of previous work,

Section Three: illustrates the methodology,

Section Four : describes the implementation steps followed in this project, and finally,

Section Five: summarizes the conclusion.

Anubhav Srivastava is a B.tech student Working in embedded systems and data science.

Ayushi Jain (B.tech)is highly skilled at image processing. She has done large number of projects and research work during herundergraduate degree.She has a keen interest in astrophysics.

\section{BACKGROUND}

Computer vision is considered study and application of methods which allows computers to examine and extract image contents or content of multidimensional data in general to facilitate solving a specific vision problem, such as pattern classification problem [2]. They add Recognition is considered one of the main six areas in computer-vision, which are sensing, preprocessing, segmentation, description, recognition, and interpretation. Heidemann (2005) has presented an approach to establish image categories automatically using histograms, colors and shape descriptors with an unsupervised learning method, Seng and Mirisaee (2009) adds that the most popular analysis techniques that have been used for both recognition and

classifications of two dimensional (2D) fruit images are color-based and shape-based analysis methods but, different fruit images may have similar color and shape values. So, using color or shape features analysis methods are still not effective enough to identify and distinguish fruits images.Rocha et. al. (2010) says that recognizing different kinds of vegetables and fruits is a recurrent task in supermarkets, where the cashier must be able to point out not only the species of a particular fruit but also its variety which will determine its price.

\section{METHODOLEGY}

The proposed method can process, analyze and recognize fruits based on color. It is often useful to simplify a monochrome problem by improving contrast or separation . In addition, it is easier and faster in processing. Our methodology can be summarized in the learning and recognition sections. (Figure 1) is the general block diagram for the project, as illustrated below, the program takes different fruit/vegetable images for the learning purpose,calculate their histograms, then, capture an image for Unknown fruit/vegetable, calculate its histogram, compare between histogram of unknown fruit image and histograms of learnt fruit images using Chi-square Method to find best matching image.Figure 2: Fruit/vegetables recognition Block Diagram

\section{IMPLEMENTATION STEPS}

\section{A. Preprocessing of Image}

\section{i. Input Images}

Input images for different fruits/vegetables, in our implementation, the application had learnt Apple,Orange, Blueberry. 
Identification of Apple, orange and berry and segregation according to sizes in images using Image processing techniques

\section{ii. Applying Gaussian Blur}

In image processing, a Gaussian blur (also known as Gaussiansmoothing) is the result of blurring an image by a Gaussian function.

\section{iii. Convert To HSV Model}

Convert images to HSV model, we converted images to HSV model; because it is the right choice for detecting objects, other color models are sensitive to illumination, and this complicating the task of detecting the fruit. In HSV model the value (V) component is still sensitive to illumination, but we skipped this component to avoid illumination effects. Arivazhagan et. al. (2010) indicate that the HSV representation is often selected for its invariant properties. The hue is invariant under the orientation of an object with respect to the illumination and camera direction and hence more suited for object retrieval. We use these equations to convert them:

For S:

$\mathrm{S}=\operatorname{Max}-\operatorname{Min} \backslash \operatorname{Min}$

Where Max is the maximum value of $(R, G, B)$ Images and Min the minimum value of $(R, G, B)$ Images.

For H:

If $\mathrm{Max}=\mathrm{Min}$ then $\mathrm{H}$ is undefined

If $\mathrm{Max}=\mathrm{R}$ and $\mathrm{G} \geq \mathrm{B}$ then $\mathrm{H}=60 *(\mathrm{G}-\mathrm{B}) /($ Max-Min)

If $\mathrm{Max}=\mathrm{R}$ and $\mathrm{G}<\mathrm{B}$ then $\mathrm{H}=60 *(\mathrm{G}-\mathrm{B}) /($ Max-Min $)+360$

If $\mathrm{Max}=\mathrm{G}$ then $\mathrm{H}=60 *(\mathrm{~B}-\mathrm{R}) /(\mathrm{Max}-\mathrm{Min})+120$

If $\operatorname{Max}=\mathrm{B}$ then $\mathrm{H}=60 *(\mathrm{R}-\mathrm{G}) /(\mathrm{Max}-\mathrm{Min})+240$

\section{iv. Masking}

Image masking is a process of graphics software like Photoshop to hide some portions of an image and to reveal some portions. It is a non-destructive process of image editing.

\section{v. Opening and closing on masked image}

Opening is just another name of erosion followed by dilation. It is useful in removing noise.Closing is reverse of Opening, Dilation followed by Erosion. It is useful in closing small holes inside the foreground objects, or small black points on the object.This processes is required to remove the external noise from the image which are in the form of black or white

Dots which can cause error in identification of fruits.

\section{A. Comparing contours}

The contour of the preprocessed image is used to calculate the area and then size of the thus identified fruit.The size comparison is then used for segregation according to size.

\section{B. Capture an image for the intended fruit to be recognized:}

After capturing the image, apply the following steps as in the learning process.

Convert image to HSV model.

To avoid the illumination effects we must convert the image to HSV model as in the learning process that mentioned previously.

Find the Histogram of this image.
Calculate the histogram of captured image in order to compare it with stored histograms of learnt images.

\section{Comparing between learnt images and captured image:}

Compare between the histogram of captured image and each of the histograms of learnt fruit images that we stored in the array of histograms using the Chi-square method [1], in general, Chi-square test is a test that uses the chi-square statistic to test the fit between a theoretical frequency distribution and a frequency distribution of observed data for which each observation may fall into one of several classes [5]. Chi-square method is considered a minimum distance classifier and can be expressed in the following equation; it gives the difference between two images.

D chi-square $(\mathrm{H} 1, \mathrm{H} 2)=\sum \mathrm{i}((\mathrm{H} 1$ (i) $-\mathrm{H} 2$ (i)) 2/ (H1 (i) $+\mathrm{H} 2$ (i)))

where $\mathrm{H} 1$ is the histogram of the learnt image, $\mathrm{H} 2$ is the histogram of captured image, it epresses the sum of squere diffrence between the value of histogram bins of learnt images and the value of histogram bins of captured image, divided by the sum of both values of histogram bins, in which a low difference between the histograms represents better match than high, and a perfect match is 0 as Bradski and Kaehler indicates.

\section{Find the matching image:}

With having the results of histograms comparisons, we compared between these results to find the least one of them, which represents the histogram of matching image, identified this image and print it out as indication of image matching.(Figure 3) depicts the implementation step by step.

Figure 3. Flow chart of the project

Taking input in the form of Video or static images

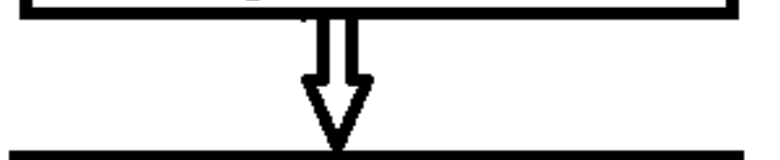

Preprocessing the image to filter any noise

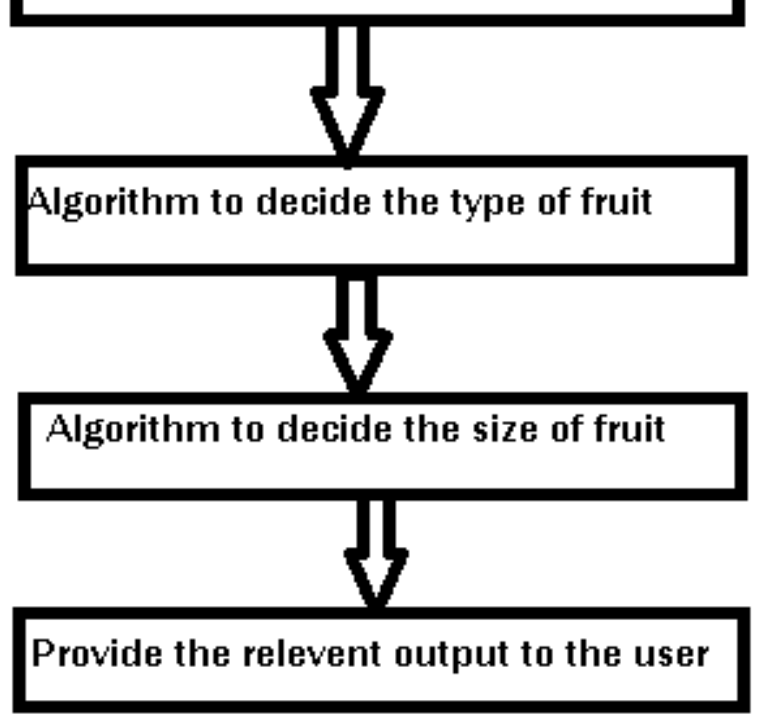


International Journal of Engineering and Technical Research (IJETR)

ISSN: 2321-0869 (O) 2454-4698 (P) Volume-8, Issue-8, August 2018

Figure : Learnt and Tested Images
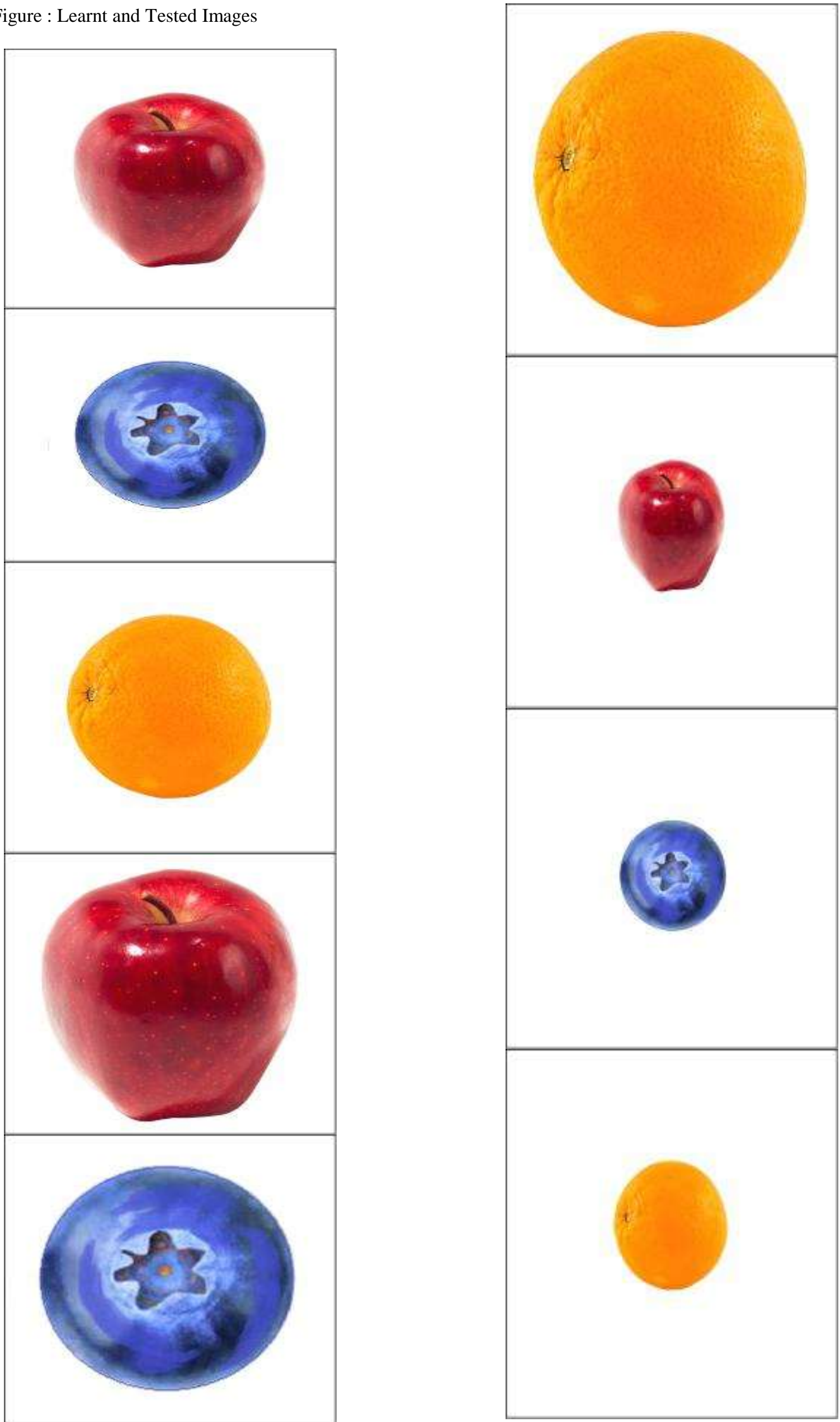
Identification of Apple, orange and berry and segregation according to sizes in images using Image processing techniques

\section{E. Code used}

import con 2

import numpy as $n p$

import os

from os.path import join, isfile

$\mathrm{cap}=\mathrm{c} / \mathrm{V}$.Videocapture $(-1)$

I_r $=n p . \operatorname{arrag}([0,100,100])$ \#red

U_ $r=n p . \operatorname{srray}([10,255,255 \mathrm{D})$ \#red

$\mathrm{I}_{-} 0=n p$.array $\left.(11,100,100]\right)$ \#orange

$U_{-} 0=n p$.array $(20,255,255 \mathrm{D})$ \#orange

I_b=np.array $(100,100,100]$ \#blue

$u_{-} b=n p$.array $(140,255,255]$ \#blue

kernel $=$ np.ones((5,5),np.uint8)

while True:

ret,img=cap read0

blur=co2. GaussianBlur(img,(7,7),0)

c/2.imshoun'gaussian', blur')

hSV $=$ cV2.cotcolor(blur, (W2.COLOR_BGR2HSV)

mask1=cw2.inRange(hsv, I_r, U_r)

min_red2=np.array $[(160,100,100]$

max_red2=np.array $(179,255,255]$

mask2=cv2. inRange(hsv, min_red2,max_red2)

mask $=m a s k 1+m a s k 2$

kernel=cw2.getstructuringElement(cw2.MORPH_ELLIPSE,(15,15))

mask_closed=cw2. morphologyex(mask, Cw2.MORPH_CLOSE, kernel)

mask_clean=cw2.morphologyex(mask_closed,cw2.MORPH_OPEN, kernel)

w2.imshom('mask_clean',mask_clean)

\#ov2.imshow'mask_closed',mask_closed) noise present in the output

\#res=cw2.bitwise_and(mask_clean,mask_clean,mask=mask)

\#gray $=$ ch2.cotcolor'mask_clean,cW2.COLOR_BGR2GRAY)

\#ret, thresh $=$ cw2.threshold(gray, 50, 255, 2)

im2, contours, hierarchy $=$

w2.findContours(mask_clean,cw2.RETR_EXTERNAL,,W2.CHAIN_APPROX_SIMPLE)

if len(contourspo:

for c in contours:

epsilon $=0.01^{*}$ crv2. arcLength(c, True $)$

\section{CONCLUSION}

This project was based on color and size through comparing image histograms to find the best matching image.Our experimental results proved that this application shows accuracy with $75 \%$ of identifying fruits, (Figure4) shows samples of learnt images and tested images.In order to improve and enhance this application, there are some future work should be implemented, providing a user friendly interface, and expanding the range of fruits known by the application will increase performance of the application.

\section{ACKNOWLEDGMENT:}

The authors are grateful to acknowledge the authors of reference papers which helped in successfull completion of research paper.

\section{REFERENCES}

[1] G. Bradski, A. Kaehler, (2008), "Learning OpenCV", O’Reilly Media, Inc.,: United States of America.
[2] W. Seng, S. Mirisaee, (2009), "A New Method for Fruits Recognition System", Electrical Engineering and Informatics, 2009. ICEEI '09. International Conference on., pp. 130-134.

[3] Rocha, A., Hauagge, D., Wainer, J., Goldenstein, S. ,(2010), “Automatic fruit and vegetable classification from images", Elsevier, pp. 1-11.

[4] Arivazhagan, S., Shebiah, R., Nidhyanandhan, S., Ganesan, L., (2010), "Fruit Recognition using Color and Texture Features", Journal of Emerging Trends in Computing and Information Sciences, 1, (2), pp. $90-94$

[5] ictionary.com,http://dictionary.reference.com/browse/chi-square+test, 6 June 2012.

[6] Heidemann, G., (2005), "Unsupervised image categorization", Image and Vision Computing 23, (10), pp. 861-876.

\section{Authors:}

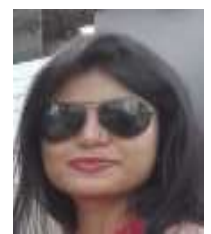

Ayushi Jain (B.tech)is highly skilled at image processing. She has done large number of projects and research work during herundergraduate degree.She has a keen interest in astrophysics.

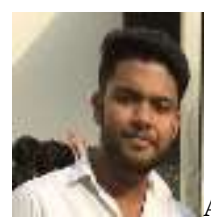

Anubhav Srivastava is a B.tech student Working in embedded systems and data science. 